\title{
The complete characterization of tangram pentagons
}

\section{Sarah Sophie Pohl ${ }^{1} \cdot$ Christian Richter $^{2}$}

Received: 17 June 2020 / Accepted: 22 August 2020 / Published online: 14 September 2020

() The Author(s) 2020

\begin{abstract}
The old Chinese puzzle tangram gives rise to serious mathematical problems when one asks for all tangram figures that satisfy particular geometric properties. All 13 convex tangram figures are known since 1942. They include the only triangular and all six quadrangular tangram figures. The families of all $n$-gonal tangram figures with $n \geq 6$ are either infinite or empty. Here we characterize all 53 pentagonal tangram figures, including 51 non-convex pentagons and 31 pentagons whose vertices are not contained in the same orthogonal lattice.
\end{abstract}

Keywords Tangram · Dissection · Tiling · Pentagon · Lattice

Mathematics Subject Classification 52C20 (Primary); 00A08 · 05B45 · 51M04

\section{Introduction}

The tangram, known as a Chinese puzzle (Goodrich 1817), is a collection of seven polygons, called tans: five isosceles right triangles, two with legs of length 1, one with $\sqrt{2}$ and two with 2 , a square with sides of length 1 and a parallelogram with sides of length 1 and $\sqrt{2}$ and an angle of $\frac{\pi}{4}$. These seven pieces are arranged, using Euclidean isometries, to form dissections of prescribed or unknown polygons, as in Fig. 1. A dissection (or tiling) of a polygon $P$ into pieces (or tiles) $P_{1}, \ldots, P_{k}$ is given if $P$ is the union of all pieces $P_{i}, 1 \leq i \leq k$, and if no two pieces have interior points

To our teachers and friends Prof. Dr. Johannes Böhm on the occasion of his 95th birthday, Prof. Dr. Eike Hertel on the occasion of his 80th birthday and Dr. Carsten Müller for 30 years of service as the school master of the Carl Zeiss Gymnasium Jena.

$凶$ Christian Richter

christian.richter@uni-jena.de

Sarah Sophie Pohl

sarah.sophie.pohl@gmail.com

1 Ringau 40, 37327 Leinefelde, Germany

2 Institute for Mathematics, Friedrich Schiller University, 07737 Jena, Germany 

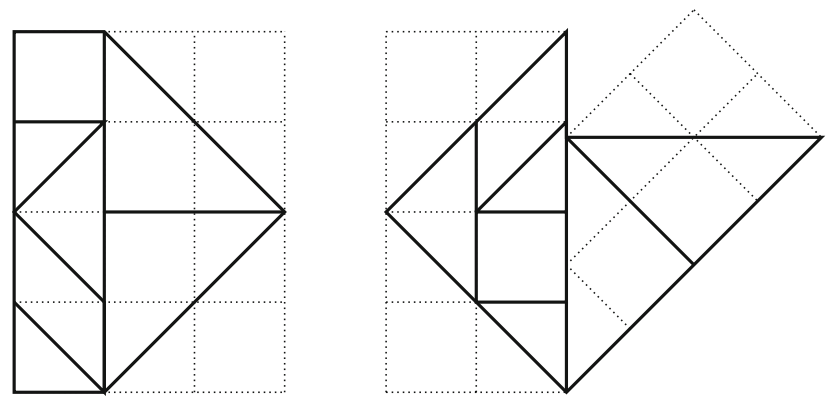

Fig. 1 The seven tans along with their lattices, dissecting a convex and a non-convex pentagon
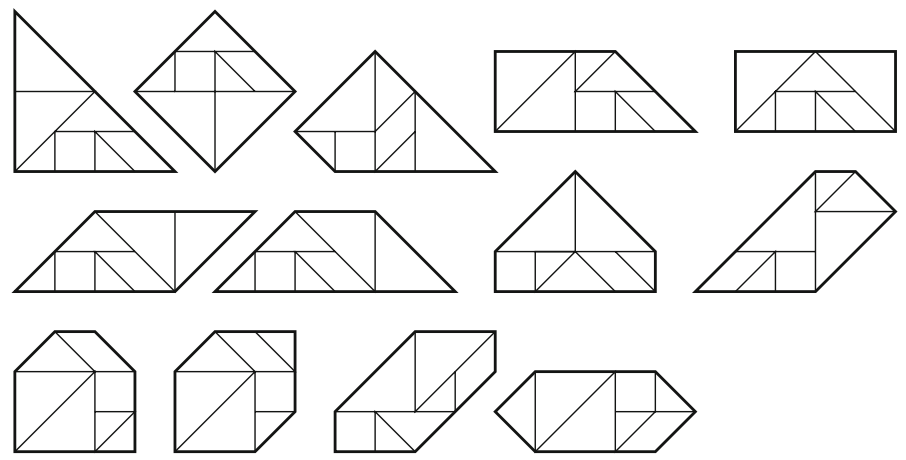

Fig. 2 All 13 convex tangrams

in common. A polygon $T$ is called a tangram if $T$ can be dissected into (isometric images of) the seven tans.

Tangram puzzles usually ask to find dissections of prescribed polygons (Elffers 1978; Goodrich 1817; Read 1985; Slocum et al. 2004). We do not address aspects of the tangram related to craft, art and design. Fruitful mathematical problems appear when one aims to detect systematically all tangrams satisfying particular geometric properties. Such questions have been posed and studied in several books (e.g. Elffers 1978; Müller 2013, Chapter 7; 2020; Read 1985; Slocum et al. 2004)), papers in mathematical journals (e.g. Gardner 1974a; Graber et al. 2016; Heinert 1998a, b; Read 2004; Wang and Hsiung 1942), private publications (Müller 1997-2014) and contributions to mathematical competitions (Brunner 2014, 2015; Heinert 1996; Pohl 2018, 2019, 2020). The most prominent result of that kind is the following one by Wang and Hsiung (cf. Fig. 2).

Theorem 1 (Wang and Hsiung 1942) There exist, up to isometry, exactly 13 convex tangrams: one triangle, six quadrangles, two pentagons and four hexagons.

This motivates the question for other natural classes of tangrams. When asking for all simple $n$-gons for fixed $n$, the cases of triangles and quadrangles appear trivial or simple, see Sect. 2. Already for hexagons one gets uncountably many incongruent tangrams: for example, one may shift the right part of the dissection on the right-hand 
side of Fig. 1 slightly up, this way obtaining a continuum of hexagons. Similarly, one finds uncountably many simple $n$-gons for all $n=7, \ldots, 23$. The number of vertices of any tangram is at most 23 , which is the total number of vertices of all seven tans.

Here we characterize all simple pentagonal tangrams, this way answering a question that seems to have been posed by Lindgren in 1968 (cf. Gardner 1974a). Most of them are non-convex, see e.g. Fig. 1. In the case of pentagons we observe a technical problem concerning the respective position of the tans: In the present paper a lattice always means an isometric image of $\mathbb{Z}^{2}$. Every tan induces a unique lattice that contains all its vertices. If a tangram admits a dissection such that all tans induce the same lattice, we call it a lattice tangram. Otherwise we call it a non-lattice tangram. Figure 1 illustrates both situations. Simple polygons that are lattice tangrams have been called snug tangrams by Read (Gardner 1974a; Read 1985, 2004).

All convex tangrams from Theorem 1 are lattice tangrams. We shall obtain the following counterpart on pentagons.

Theorem 2 There exist, up to isometry, exactly 53 simple pentagons that are tangrams: two convex ones, 20 non-convex lattice ones and 31 non-convex non-lattice ones.

These pentagons are given in detail in Sect. 4. Before that, we comment briefly the situation for triangular and quadrangular tangrams (Sect. 2), and we provide a topological tool that permits a systematic approach to pentagons (Sect. 3).

The present paper is an extended version of Pohl (2019).

\section{Triangular and quadrangular tangrams}

Proposition 3 There exist, up to isometry, only one triangular and six quadrangular tangrams. All of them are convex.

Before proving Proposition 3, we note two obvious properties of tangrams.

Lemma 4 (i) The area of every tangram is 8.

(ii) The sizes of the inner angles of every tangram are integer multiples of $\frac{\pi}{4}$.

Proof of Proposition 3 By Lemma 4, only the isosceles right triangle with legs of length 4 can be a triangular tangram. This triangle is indeed a tangram (cf. Wang and Hsiung 1942 or Fig. 2).

All six convex quadrangular tangrams are known from Wang and Hsiung (1942), see Fig. 2. It remains to show that there are no non-convex quadrangular tangrams. For that, assume that we are given such a quadrangle $Q$. Since its inner angles sum up to $2 \pi$ and satisfy Lemma 4(ii), their sizes must be $\frac{\pi}{4}, \frac{\pi}{4}, \frac{\pi}{4}$ and $\frac{5 \pi}{4}$. Let $\xi, \eta \in \mathbb{R}$ be the lengths of the sides not emanating from the non-convex vertex, see Fig. 3.

We have, w.l.o.g.,

$$
\xi \geq \eta>\frac{\xi}{\sqrt{2}}
$$




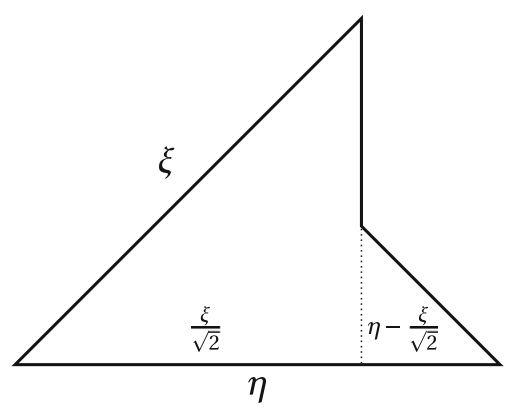

Fig. 3 A potential non-convex quadrangular tangram

Lemma 4(i) together with (1) gives

$8=\frac{1}{2}\left(\frac{\xi}{\sqrt{2}}\right)^{2}+\frac{1}{2}\left(\eta-\frac{\xi}{\sqrt{2}}\right)^{2}\left\{\begin{array}{l}>\frac{1}{2}\left(\frac{\xi}{\sqrt{2}}\right)^{2}=\frac{1}{4} \xi^{2}, \\ \leq \frac{1}{2}\left(\frac{\xi}{\sqrt{2}}\right)^{2}+\frac{1}{2}\left(\xi-\frac{\xi}{\sqrt{2}}\right)^{2}=\left(\frac{2-\sqrt{2}}{2}\right) \xi^{2}\end{array}\right.$

The two inequalities give

$$
5.226 \cdots=\sqrt{8(2+\sqrt{2})} \leq \xi<4 \sqrt{2}=5.656 \ldots
$$

Since $\xi$ and $\eta$ are sums of side lengths of tans, which are integers or integer multiples of $\sqrt{2}$, we obtain

$$
\xi, \eta \in\{k+l \sqrt{2}: k, l \in\{0,1, \ldots\}\}
$$

Then (3) implies

$$
\xi \in\{4+\sqrt{2}, 1+3 \sqrt{2}\}
$$

For any of the two choices of $\xi$, we see that the left-hand equation from (2) does not have a solution $\eta$ that satisfies (4).

\section{A topological lemma}

Now we work in a slightly generalized setting. An isosceles right triangle with legs of length 1 is called a basic triangle. A polygon admitting a dissection into finitely many basic triangles is called a generalized tangram. Of course, every tangram is a generalized tangram, since every tan can be subdivided into basic triangles (see the left-hand part of Fig. 4). Although that subdivision is not unique (namely, for the square tan as well as for the large triangular tans), the lattice associated to every basic 

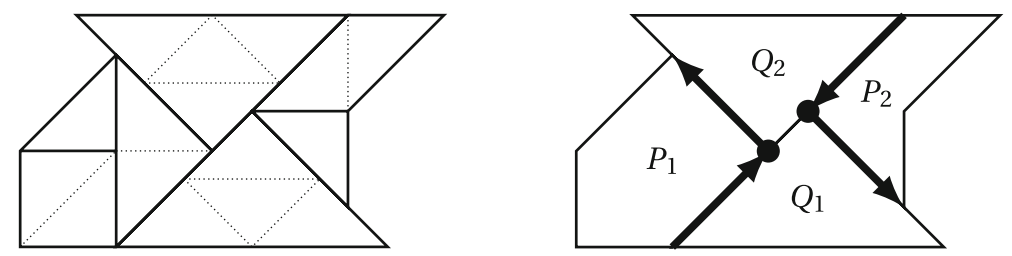

Fig. 4 A tangram with a subdivision into basic triangles (left) and concepts for the proof of Lemma 5 (right)

triangle coincides with that of the original tan. So the concepts of lattice and non-lattice tangrams extend naturally to generalized tangrams.

In the remainder of this section we shall prove the following lemma. It will be a crucial tool for the classification of all pentagonal tangrams, since these are either convex or possess exactly one non-convex vertex (cf. Sect. 4).

Lemma 5 Let $T$ be a generalized tangram that is a simple polygon having exactly one non-convex vertex $v_{1}$. Then one of the following is satisfied.

(I) All vertices of $T$ belong to the same lattice $\Lambda=\varphi\left(\mathbb{Z}^{2}\right)$, where $\varphi$ is a Euclidean isometry. Every side of $T$ is parallel to one of the segments $\varphi((0,0)(1,0))$, $\varphi((0,0)(0,1)), \varphi((0,0)(1,1))$ or $\varphi((0,0)(1,-1))$.

(II) One of the straight lines defined by a side of $T$ emanating from $v_{1}$ dissects $T$ into two convex generalized tangrams $T_{1}$ and $T_{2}$ with corresponding lattices $\Lambda_{1}$ and $\Lambda_{2}$ as in (I). The lattice $\Lambda_{2}$ is the image of $\Lambda_{1}$ under some rotation by $\frac{\pi}{4}$.

\subsection{Preparations}

Suppose now that we are given a generalized tangram $T$ satisfying the assumptions of Lemma 5, along with a dissection into basic triangles. Two basic triangles are called equally oriented if their associated lattices differ at most by a translation. Since $T$ is a simple polygon, there are at most two different orientations. Let $P$ be the union of one class of equally oriented basic triangles, and let $Q$ be the union of the remaining basic triangles. Then $Q$ is either empty or the lattices associated to the pieces of $Q$ are obtained from those of $P$ by rotations of $\frac{\pi}{4}$. Two basic triangles of $P$ are considered equivalent if their lattices agree. Let $P_{1}, \ldots, P_{m}$ be the respective unions of all classes of equivalent basic triangles of $P$. Similarly, $Q$ splits into unions $Q_{1}, \ldots, Q_{n}$ of equivalent triangles. This way we obtain the dissection

$$
T=\left(P_{1} \cup \cdots \cup P_{m}\right) \cup\left(Q_{1} \cup \cdots \cup Q_{n}\right) .
$$

(Figure 4 illustrates an eight-angled tangram with corresponding dissections into tans as well as into basic triangles, on the left, and the resulting dissection introduced above, on the right.)

We cannot assume the polygons $P_{i}$ and $Q_{j}$ to be simple or even connected. In that setting we call an element $v$ of the boundary $\operatorname{bd}(R)$ of a polygon $R$ a vertex of $R$ if there is no circular disc $D$ centered at $v$ such that $D \cap R$ is a half-disc of $D$. When 

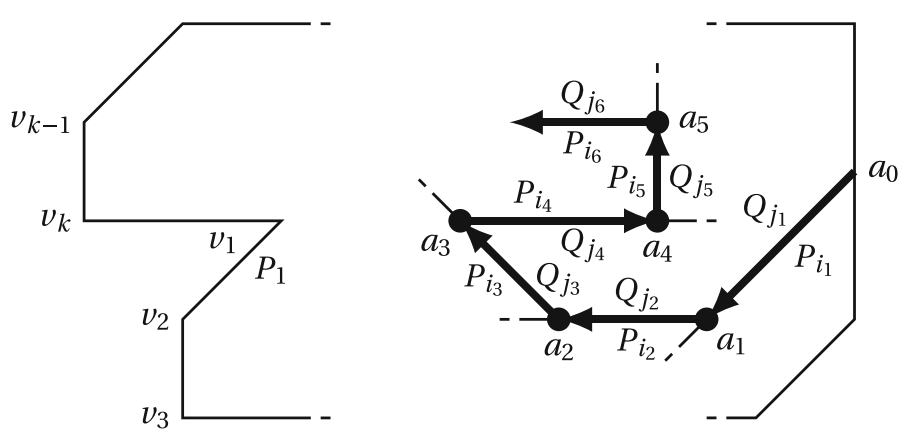

Fig. 5 The vertices of $T$ and the arc $\Delta$

speaking of a side of $R$, we mean a maximal line segment in $\operatorname{bd}(R)$ that does not contain one of the vertices of $R$ in its relative interior.

Fact 6 Let $1 \leq i<i^{\prime} \leq m$ and $1 \leq j<j^{\prime} \leq n$. Then

(i) $P_{i}$ and $P_{i^{\prime}}$ (and, similarly, $Q_{j}$ and $Q_{j^{\prime}}$ ) do not have a common vertex,

(ii) $P_{i}$ and $Q_{j}$ have at most one common vertex.

Proof (i): A common vertex of $P_{i}$ and $P_{i^{\prime}}$ would belong to both the lattices associated to $P_{i}$ and $P_{i^{\prime}}$, respectively. But this intersection is empty, because these lattices do not agree and are translates of each other.

(ii): The lattices associated to $P_{i}$ and $Q_{j}$ are images of each other under a rotation by $\frac{\pi}{4}$. Thus they have at most one point in common, the only possible joint vertex of $P_{i}$ and $Q_{j}$.

The skeleton $S$ of the dissection in (5) is the union of the boundaries of its pieces, i.e.,

$$
S=\operatorname{bd}\left(P_{1}\right) \cup \cdots \cup \operatorname{bd}\left(P_{m}\right) \cup \operatorname{bd}\left(Q_{1}\right) \cup \cdots \cup \operatorname{bd}\left(Q_{n}\right) .
$$

We shall deal with arcs contained in $S$. We say that an arc starts (or ends) in $P_{i}$ if a segment of positive length of the beginning (or the end) of that arc is contained in $\operatorname{bd}\left(P_{i}\right)$.

Fact 7 Let $\Theta \subseteq \operatorname{bd}(T) \cap P$ be an arc that does not contain the non-convex vertex $v_{1}$ in its relative interior. If $\Theta$ starts in $P_{i}, 1 \leq i \leq n$, then $\Theta \subseteq P_{i}$. (The analogue applies to arcs in $\operatorname{bd}(T) \cap Q$.)

Proof Assume that $\Theta \nsubseteq P_{i}$. Then there is a point $x_{0} \in \Theta$ where $\Theta$ switches from $P_{i}$ into some $P_{i^{\prime}}$ with $i^{\prime} \neq i$. Since $\Theta$ does not pass through the only non-convex vertex $v_{1}$ of $T$, the point $x_{0}$ must be a common vertex of $P_{i}$ and $P_{i^{\prime}}$. This contradicts Fact 6(i).

Let $v_{1}, \ldots, v_{k}$ be the vertices of $T$ appearing along its boundary in counterclockwise direction. These are illustrated in the left-hand part of Fig. 5. 


\section{Fact 8 From now on we can assume that}

(i) the arc $v_{1} v_{2} \ldots v_{k}$ starts in $P_{1}$ and $v_{1}$ is a vertex of $P_{1}$,

(ii) $\operatorname{bd}(T) \nsubseteq P$ and $\mathrm{bd}(T) \nsubseteq Q$.

Proof (i): There exists one of the pieces $P_{i}, 1 \leq i \leq m$, or $Q_{j}, 1 \leq j \leq n$, such that $v_{1}$ is a vertex of that piece and one of the segments $v_{1} v_{2}$ or $v_{1} v_{k}$ starts in that piece. By possibly renaming the pieces in (5) we can assume that that piece is $P_{1}$. By possibly reversing the order of vertices of $T$ we can assume that $v_{1} v_{2}$ starts in $P_{1}$, w.l.o.g.

(ii): If $\operatorname{bd}(T) \subseteq P$, then application of Fact 7 to the arc $\Theta=v_{1} v_{2} \ldots v_{k}$, which starts in $P_{1}$ by (i), yields $\Theta \subseteq P_{1}$. Consequently, all vertices $v_{1}, \ldots, v_{k}$ of $T$ are vertices of $P_{1}$, which belong to the same lattice according to the definition of $P_{1}$. This way we reach claim (I) of Lemma 5, and the proof is complete if $\operatorname{bd}(T) \subseteq P$. Thus the case $\operatorname{bd}(T) \subseteq P$ does not require further consideration.

The situation $\operatorname{bd}(T) \subseteq Q$ does not appear, since $v_{1} v_{2}$ starts in $P_{1}$ by (i).

\subsection{The arc $\Delta$}

We call an interior point $x$ of $T$ a T-vertex of the dissection (5) if $x$ is a joint vertex of two pieces $P_{i}$ and $Q_{j}$ and belongs to the relative interior of a side of some $P_{i^{\prime}}$, $i^{\prime} \neq i$, or $Q_{j^{\prime}}, j^{\prime} \neq j$. That is, a side of $P_{i^{\prime}}$ or $Q_{j^{\prime}}$ passes through $x$ and at least one joint segment of $P_{i}$ and $Q_{j}$ emanates from $x$ and cannot be continued over $x$ within the skeleton $S$. Then we say that $P_{i}$ and $Q_{j}$ are below the T-vertex $x$ and $P_{i^{\prime}}$ (or $Q_{j^{\prime}}$, respectively) is above $x$. (Note that the name T-vertex is motivated by the shape of the capital letter $\mathrm{T}$, but not by the name $T$ of our polygon.)

A directed line segment $x y \subseteq S$ is called a primal segment if there exist $i$ and $j$ such that $x y \subseteq P_{i} \cap Q_{j}, x$ is a joint vertex of $P_{i}$ and $Q_{j}, y$ is a vertex of one of $P_{i}$ or $Q_{j}$, and $x y$ does not contain further vertices of $P_{i}$ or $Q_{j}$. Figure 4 illustrates all $\mathrm{T}$-vertices as small circles and all primal segments as arrows.

Fact 9 Let $x y \subseteq P_{i} \cap Q_{j}$ be a primal segment. Then either $y=v_{1}$ and $v_{1}$ is in the relative interior of some side of one of $P_{i}$ or $Q_{j}$ or y is a T-vertex having one of $P_{i}$ or $Q_{j}$ above.

Proof Suppose that $y$ is a vertex of $P_{i}$, w.l.o.g. By Fact 6(ii), $y$ is no vertex of $Q_{j}$. So $y$ is in the relative interior of a side of $Q_{j}$.

If $y$ is in the interior of $T$, Fact 6 implies that $y$ is a vertex of some $Q_{j^{\prime}}, j^{\prime} \neq j$. Then $y$ is a T-vertex with $P_{i}$ and $Q_{j^{\prime}}$ below and $Q_{j}$ above.

Now let $y \in \operatorname{bd}(T)$. If $y \neq v_{1}$ then $y$ had to be be a vertex of both $P_{i}$ and $Q_{j}$, since $v_{1}$ is the only non-convex vertex of $T$. This contradiction shows that $y=v_{1}$.

Next we define a particular arc $\Delta=a_{0} a_{1} \ldots a_{l}$ in the skeleton $S$ as a union of primal segments (see Fig. 5). We know from Fact 8 that, when starting in $v_{1}$ and following $\operatorname{bd}(T)$ counter-clockwise, we meet a first point $a_{0} \in \operatorname{bd}(T) \backslash\left\{v_{1}\right\}$ where we switch from $P$ to $Q$. There exist (by Fact 6(i) unique) $1 \leq i_{1} \leq m$ and $1 \leq j_{1} \leq n$ such that $a_{0}$ is a vertex of both $P_{i_{1}}$ and $Q_{j_{1}}$. There is (at least) one primal segment $a_{0} a_{1} \subseteq P_{i_{1}} \cap Q_{j_{1}}$ such that $P_{i_{1}}$ and $Q_{j_{1}}$ are left and right beside $a_{0} a_{1}$, respectively. This segment is the beginning of $\Delta$. 
Now we continue the definition of $\Delta$ recursively. Suppose that $a_{r-1} a_{r} \subseteq P_{i_{r}} \cap Q_{j_{r}}$ is the last chosen segment in $\Delta$. By Fact 9 , we can continue as follows.

- If $a_{r}=v_{1}$, we put $l=r$ and $\Delta$ is complete.

- If $a_{r}$ is a T-vertex with $P_{i_{r}}$ above, there is a (by Fact 6(i) unique) $1 \leq i_{r+1} \leq m$ such that $P_{i_{r+1}}$ is below $a_{r}$. We put $j_{r+1}=j_{r}$ and continue $\Delta$ with a next primal segment $a_{r} a_{r+1} \subseteq P_{i_{r+1}} \cap Q_{j_{r+1}}$ having $P_{i_{r+1}}$ on the left and $Q_{j_{r+1}}$ on the right.

- If $a_{r}$ is a T-vertex with $Q_{j_{r}}$ above, we proceed analogously and obtain a next primal segment $a_{r} a_{r+1} \subseteq P_{i_{r+1}} \cap Q_{j_{r+1}}$ having $P_{i_{r+1}}$ on the left and $Q_{j_{r+1}}$ on the right, where $i_{r+1}=i_{r}$.

Note that this definition terminates after finitely many steps, since it produces no loops. Indeed, suppose that $x_{0}$ would be a first point of self-intersection. Then $x_{0}$ cannot be in the relative interior of a primal segment, because these do not contain vertices. Hence $x_{0}$ is a T-vertex with $x_{0}=a_{r}=a_{r^{\prime}}, r<r^{\prime}$, and the primal segments $a_{r-1} a_{r}$ and $a_{r^{\prime}-1} a_{r^{\prime}}$ arrive at $x_{0}$ from opposite directions. This is impossible, because both segments have $P$ on the left and $Q$ on the right.

In the following we use the notation $\widehat{x y}, x, y \in \operatorname{bd}(T)$, for the counter-clockwise arc in $\operatorname{bd}(T)$ that begins in $x$ and ends in $y$.

Fact 10 When following $\operatorname{bd}(T)$ counter-clockwise, $a_{0}$ is the only point where one switches from $P$ to $Q$. Accordingly, there exists exactly one point $b_{0} \in \widehat{a_{0} v_{1}} \backslash\left\{a_{0}\right\}$ where one switches back from $Q$ to $P$.

Proof First note that we can recover $\Delta$ uniquely by the following backwards procedure: Applying Fact 9 to the last segment $a_{l-1} a_{l}=a_{l-1} v_{1}$ of $\Delta$, we see that $v_{1}$ is in the relative interior of a side of one of $P_{i_{l}}$ or $Q_{j_{l}}$. Since there is at most one of the $m+n$ tiles from (5) having $v_{1}$ in the relative interior of one of its sides, this determines that very tile uniquely. Moreover, since $P$ is on the left and $Q$ is on the right of $\Delta$, this recovers at least a little segment at the end of $\Delta$. Now we find $a_{l-1}$ by following that direction until we end at a T-vertex (or at $\operatorname{bd}(T)$, where we meet $a_{0}$ ). The polygon above that T-vertex $a_{l-1}$ is $P_{i_{l-1}}$ or $Q_{j_{l-1}}$. Again by using the lateral position of $P$ and $Q$ relative to $\Delta$ we determine the direction of the segment $a_{l-2} a_{l-1}$. Following that direction until the next T-vertex (or until bd(T), where we find $a_{0}$ ) we find $a_{l-2}$. Continuing this way we reproduce $\Delta$ uniquely.

Now, for proving the fact, let $a_{0}^{\prime} \in \operatorname{bd}(T)$ be a point where one switches from $P$ to $Q$. Starting from $a_{0}^{\prime}$, we define an arc $\Delta^{\prime} \subseteq S$ from $a_{0}^{\prime}$ to $v_{1}$ by the same rules as we did with $\Delta$ from $a_{0}$. We can reproduce $\Delta^{\prime}$ beginning at its end $v_{1}$, as we did with $\Delta$. But this reproduction gives the same arc $\Delta^{\prime}=\Delta$, so that $a_{0}^{\prime}=a_{0}$.

Fact 11 Let $R_{0}$ be the piece among $P_{1}, \ldots, P_{m}, Q_{1}, \ldots, Q_{n}$ such that the segment $v_{k} v_{1}$ ends in $R_{0}$. Then $v_{1}$ is in the relative interior of a side of $R_{0}$.

Proof Assume that our claim fails. Then $v_{1}$ is a vertex of $R_{0}$.

Case 1: $R_{0}=P_{i^{*}}$ for some $1 \leq i^{*} \leq m$. Fact 6(i) yields $i^{*}=1$, since $v_{1}$ is a vertex of both $P_{1}$ and $P_{i^{*}}$. Applying Fact 7 to the arcs $\widehat{v_{1} a_{0}}, \widehat{b_{0} v_{1}} \subseteq P$, we see that $a_{0}$ and $b_{0}$ are vertices of $P_{1}$. Similarly, applying Fact 7 to $a_{0} b_{0} \subseteq Q$, we see that both $a_{0}$ and $b_{0}$ are vertices of some $Q_{j}$. This contradicts Fact 6(ii). 

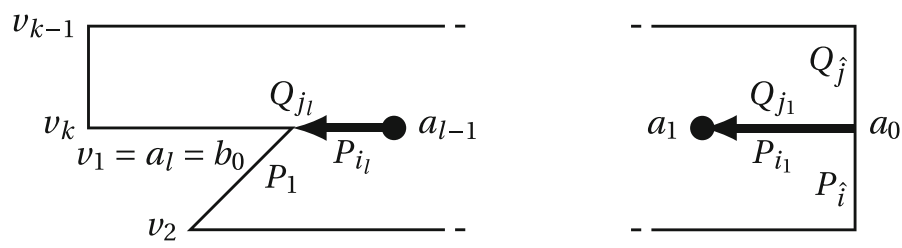

Fig. 6 The situation after Fact 12

Case 2: $R_{0}=Q_{j^{*}}$ for some $1 \leq j^{*} \leq n$. Now $b_{0}=v_{1}$, since we switch from $Q_{j *}$ to $P_{1}$ in $v_{1}$. Applications of Fact 7 to $\widehat{v_{1} a_{0}} \subseteq P$ and $\widehat{a_{0} v_{1}} \subseteq Q$ shows that $v_{1}$ and $a_{0}$ are joint vertices of both $P_{1}$ and $Q_{j^{*}}$, again contradicting Fact 6(ii).

Now we learn more on the end of $\Delta=a_{0} a_{1} \ldots a_{l}$.

Fact 12 The points $a_{l-1}, a_{l}=v_{1}$ and $v_{k}$ are collinear. The vertex $v_{1}$ is in the relative interior of a side of $Q_{j_{l}}$. In particular, $b_{0}=v_{1}$.

Proof There is at most one piece among $P_{1}, \ldots, P_{m}, Q_{1}, \ldots, Q_{n}$ having $v_{1}$ in the relative interior of a side. By Fact 11 , such a piece $R_{0}$ exists and $v_{1} v_{k}$ represents the direction of that side. On the other hand, application of Fact 9 to the last primal segment $a_{l-1} a_{l}=a_{l-1} v_{1}$ of $\Delta$ shows that one of $P_{i_{l}}$ or $Q_{j_{l}}$ has $v_{1}$ in the relative interior of a side. Consequently, $R_{0} \in\left\{P_{i_{l}}, Q_{j_{l}}\right\}$ and $a_{l-1}, a_{l}=v_{1}$ and $v_{k}$ are collinear. Since $P_{i_{l}}$ is on the left of $a_{l-1} a_{l}$ and since $v_{k} v_{1}$ ends in $R_{0}$, we obtain $R_{0}=Q_{j_{l}}$. Finally, since $v_{k} v_{1}$ ends in $R_{0}=Q_{j_{l}}$, Fact 10 yields $b_{0}=v_{1}$.

Figure 6 gives a scheme of we have reached so far.

\subsection{Conclusion}

The counter-clockwise arcs $\widehat{v_{1} a_{0}}, \widehat{a_{0} v_{1}} \subseteq \operatorname{bd}(T)$ are contained in $P$ and $Q$, respectively. Thus there are $1 \leq \hat{i} \leq m, 1 \leq \hat{j} \leq n$ such that $\overline{v_{1} a_{0}}$ ends in $P_{\hat{i}}$ and $\overline{a_{0} v_{1}}$ begins in $Q_{\hat{j}}$. Since $a_{0}$ is a vertex of $P_{\hat{i}}, P_{i_{1}}, Q_{\hat{j}}$ and $Q_{j_{1}}$, Fact 6(i) yields $P_{\hat{i}}=P_{i_{1}}$ and $Q_{\hat{j}}=Q_{j_{1}}$. Similarly, $P_{i_{l}}=P_{1}$, because of the joint vertex $a_{l}=v_{1}$. Moreover, we apply Fact 7 to $\widehat{v_{1} a_{0}} \subseteq P$ and $\widehat{a_{0} v_{1}} \subseteq Q$ and obtain

$$
\widehat{v_{1} a_{0}} \subseteq P_{i_{1}}=P_{\hat{i}}=P_{1}=P_{i_{l}}, \quad \widehat{a_{0} v_{1}} \subseteq Q_{j_{1}}=Q_{\hat{j}}=Q_{j_{l}}
$$

In particular, both $a_{0}$ and $a_{l-1}$ are joint vertices of $P_{i_{1}}=P_{i_{l}}$ and $Q_{j_{1}}=Q_{j_{l}}$. Then Fact 6(ii) implies $a_{0}=a_{l-1}$, and in turn $l=1$. That is, the arc $\Delta$ consists of the single segment $\Delta=a_{0} a_{1}=a_{0} v_{1}$.

We see that $\Delta$ dissects $T$ along the straight line spanned by $v_{1} v_{k}$ into two convex generalized tangrams. By (6), all vertices of one of them are vertices of $P_{i_{1}}$ and all vertices of the other one are vertices of $Q_{j_{1}}$. This completes the proof of Lemma 5 . 


\section{The complete list of pentagonal tangrams}

In this section we prove Theorem 2. We distinguish the cases of convex and non-convex tangrams, the latter case being split into the two alternatives according to Lemma 5.

\subsection{Convex pentagons}

Proposition 13 There exist, up to isometry, exactly two convex pentagonal tangrams.

This follows from Theorem 1, see Fig. 2 for an illustration.

\subsection{Non-convex lattice pentagons}

Let a simple non-convex pentagon $T$ be a tangram. Since the sizes of its inner angles are integer multiples of $\frac{\pi}{4}$ (see Lemma 4(ii)) and add up to $3 \pi, T$ has only one nonconvex vertex. Thus $T$ satisfies one of the situations (I) or (II) from Lemma 5. Here in Sect. 4.2 we assume that (I) applies.

The following discussion seems to go back to Read (published in Gardner 1974a) and, independently, to Heinert (Heinert 1996). We reproduce it here, since the publication (Gardner 1974a) misses six of the 20 solutions (see (Gardner 1974b, p. 125)) and since Heinert's manuscript (Heinert 1996) is almost inaccessible. Note that Read and Heinert assumed their tangrams a priorily to be lattice tangrams. We use only the situation of Lemma 5(I), i.e., that the boundary of $T$ is arranged along a lattice.

The inner angles of $T$ have sizes $\alpha_{i}=k_{i} \frac{\pi}{4}$ with $k_{1} \in\{5,6,7\}$ (non-convex vertex) and $k_{i} \in\{1,2,3\}, 2 \leq i \leq 5$ (convex vertices). Their sum is $\alpha_{1}+\ldots+\alpha_{5}=3 \pi$, i.e., $k_{1}+\cdots+k_{5}=12$. Hence the multi-set $\left\{k_{1}, \ldots, k_{5}\right\}$ is one of $\{5,3,2,1,1\}$, $\{5,2,2,2,1\},\{6,3,1,1,1\},\{6,2,2,1,1\}$ or $\{7,2,1,1,1\}$.

For fixed $\left\{k_{1}, \ldots, k_{5}\right\}$, the permutations $k_{\varrho(1)} \ldots k_{\varrho(5)}$ give the successive order of the angles along the boundary of $T$. Cyclic shifts and reversions can be considered equal, since they correspond to isometric images of $T$. Thus it remains to consider the following 16 orders of inner angles: 53211, 53121, 53112, 52311, 52131, 51321, 52221, 52212, 63111, 61311, 62211, 62121, 62112, 61221, 72111 and 71211.

Next, for each of the 16 possible orders $k_{\varrho(1)} \ldots k_{\varrho(5)}$, one generates all corresponding pentagons that satisfy condition (I) from Lemma 5 and whose area is 8 according to Lemma 4(i). This can be done in the lattice $\mathbb{Z}^{2}$. One fixes a first vertex and the direction of the first side of $T$ : either $(1,0)$ or $(1,1)$, both have to be considered separately. Then the order $k_{\varrho(1)} \ldots k_{\varrho(5)}$ determines $T$ up to three integer parameters, since three side lengths fix $T$ up to isometry and the length of the $i$ th side is either $s_{i}$ or $s_{i} \sqrt{2}$ with $s_{i} \in\{1,2, \ldots\}$. Now one can easily find the choices of side lengths that give polygons $T$ of area 8.

(Alternatively, one could find these non-convex pentagons $T$ of area 8 as follows: One generates all convex generalized lattice tangrams of areas less than 8 , as it has been done in Wang and Hsiung (1942) for those of area 8. Then one examines for all pairs of total area 8 if and how they can be put together to form a non-convex lattice pentagon.) 


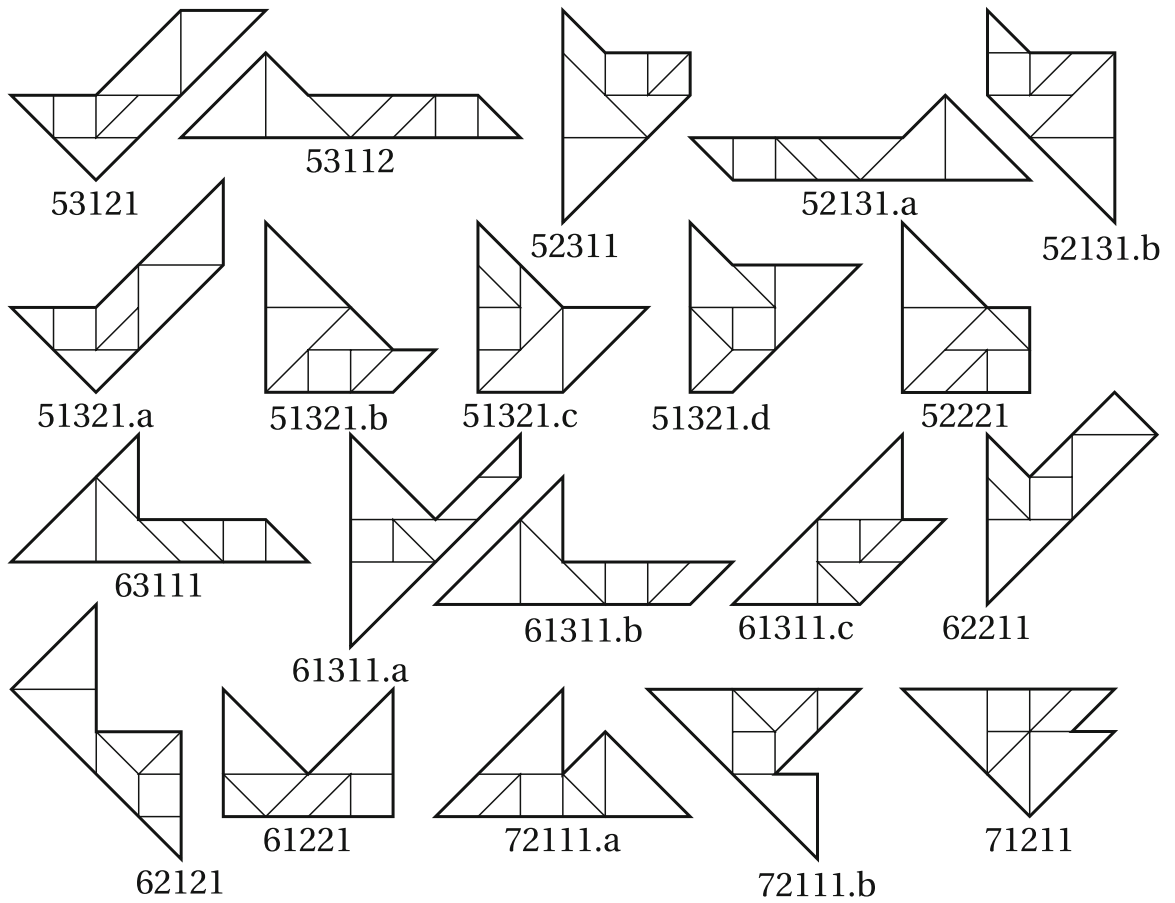

Fig. 7 All 20 non-convex pentagonal lattice tangrams (Heinert 1996)

Finally, one checks for all these resulting pentagons $T$ of area 8 if they admit a dissection into the seven tans. In fact, it turns out that such a candidate $T$ cannot be dissected only if the two large tans cannot be packed simultaneously inside $T$.

This search results in the 20 tangrams depicted in Fig. 7.

Proposition 14 (Heinert 1996) There exist, up to isometry, exactly 20 non-convex simple pentagons that are lattice tangrams.

\subsection{Non-convex non-lattice pentagons}

Now we consider non-convex pentagonal tangrams $T$ that are in the situation of Lemma 5(II). Then the straight line spanned by one of the sides emanating from the non-convex vertex dissects $T$ into two generalized tangrams $T_{1}$ and $T_{2}$. The endpoint of the dissecting segment is a joint vertex of $T_{1}$ and $T_{2}$, and it is their only joint vertex, since the lattices associated to the vertices of $T_{1}$ and $T_{2}$ share at most one point. Hence the number of vertices of $T$ is the sum of those of $T_{1}$ and $T_{2}$ minus 1 if that joint vertex is a vertex of $T$, too, or that sum minus 2 if the joint vertex is in the relative interior of a side of $T$. We obtain the following.

Fact 15 W.l.o.g., each side of any tan in $T$ has one of the directions $(1,0),(1,1)$, $(0,1)$ or $(-1,1)$. The pentagon $T$ (together with its dissection into tans) splits into two convex generalized tangrams $T_{1}$ and $T_{2}$. The vertices of $T_{1}$ belong to the lattice 


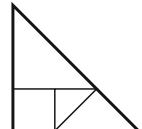

A [9]

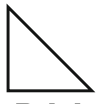

B [4]

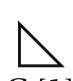

C [1]

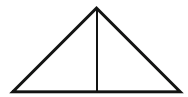

D1 [8]

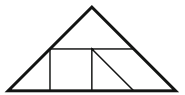

D2 [8]

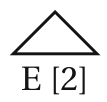

Fig. 8 All triangular generalized lattice tangrams of area less than 8 with particular dissections into tans

3311:

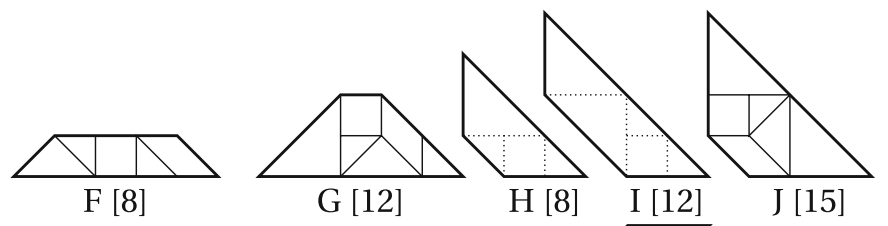

3131:

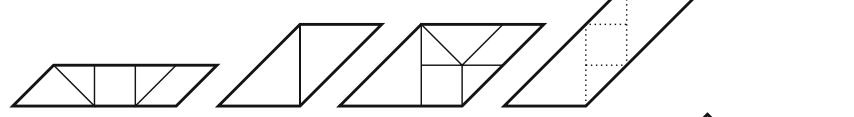

$\mathrm{K}$ [8]

$\mathrm{L}[8] \quad \mathrm{M}[12]$

$\mathrm{N}[12]$

3221:

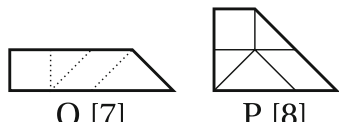

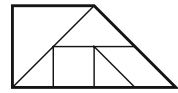

$\mathrm{Q}$ [12]

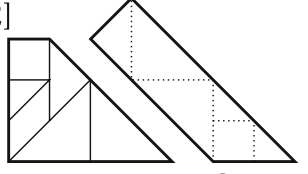

R [15]

3212:
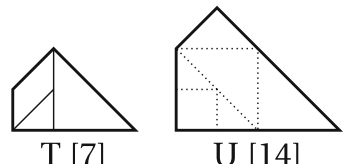

2222:
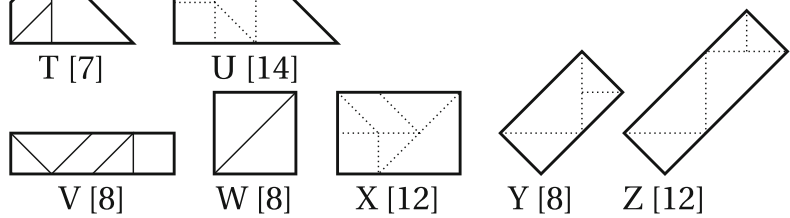

Fig. 9 All convex quadrangular generalized lattice tangrams of areas $\frac{7}{2}, 4,6,7$ or $\frac{15}{2}$ that can be dissected into a subfamily of all seven tans

$\mathbb{Z}^{2}$, the vertices of $T_{2}$ belong to an image of $\mathbb{Z}^{2}$ under a rotation by $\frac{\pi}{4}$. The polygons $T_{1}$ and $T_{2}$ have exactly one vertex $v_{0}$ in common. If their angles at $v_{0}$ add up to $\pi$ then $T_{1}$ is a quadrangle and $T_{2}$ a triangle. Otherwise the sum of that angles is smaller than $\pi$ and both $T_{1}$ and $T_{2}$ are triangles.

The area of the triangle $T_{2}$ is larger than 0 and smaller than 8 . All possible generalized tangrams with that property are illustrated in Fig. 8. There they are arranged within the lattice $\mathbb{Z}^{2}$. In square brackets we give the number of basic triangles that form the respective polygon, i.e., twice the area of that polygon. Figure 8 depicts only those dissections into tans that will be used in the sequel.

Since the areas of $T_{1}$ and $T_{2}$ sum up to 8 , the area of $T_{1}$ must be one of $\frac{7}{2}, 4,6,7$ or $\frac{15}{2}$. In other words, that area is represented by $7,8,12,14$ or 15 basic triangles. Thus the only possible triangle representing $T_{1}$ can be D1 (or D2) from Fig. 8.

Next we obtain all quadrangular candidates for $T_{1}$. The sizes $k_{1} \frac{\pi}{4}, k_{2} \frac{\pi}{4}, k_{3} \frac{\pi}{4}$ and $k_{4} \frac{\pi}{4}$ of the successive inner angles of $T_{1}$ are given by the string $k_{1} k_{2} k_{3} k_{4} \in$ 


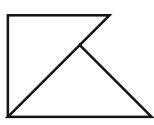

D1.D2.a

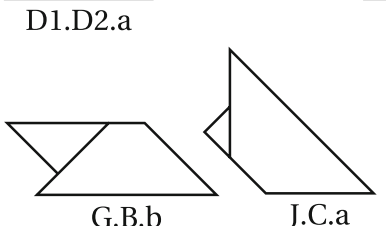

G.B.b

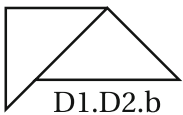

D1.D2.b

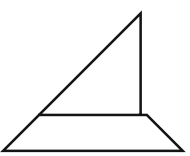

F.D1.a

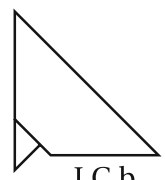

J.C.b

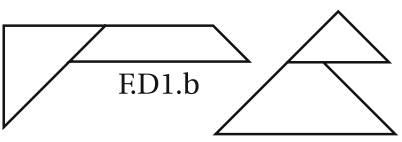

G.B.a
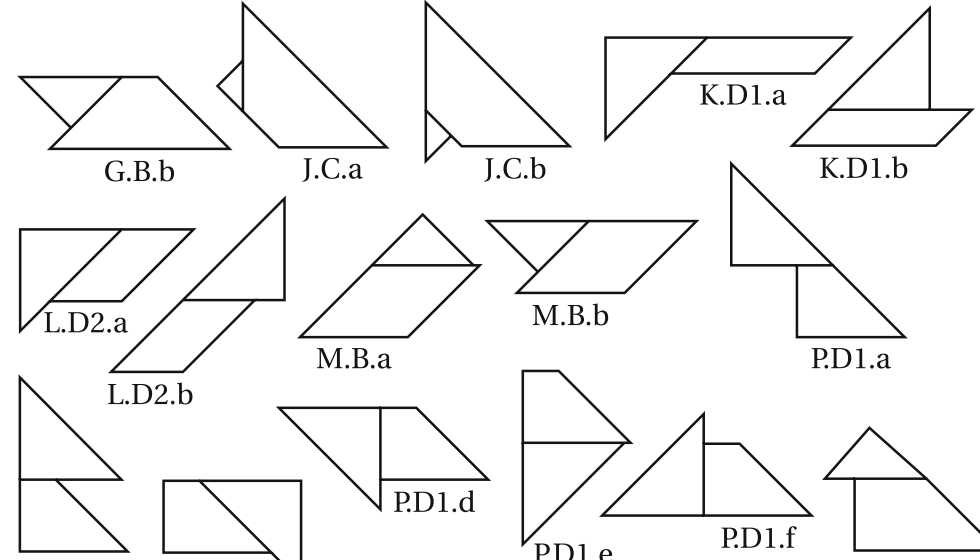

D2.b
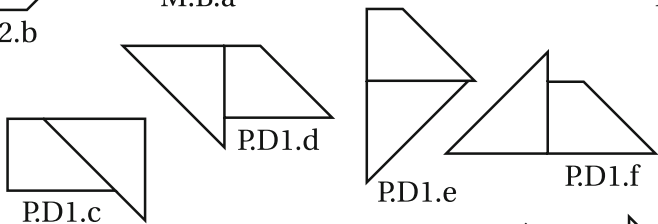

P.D1.b

P.D1.C
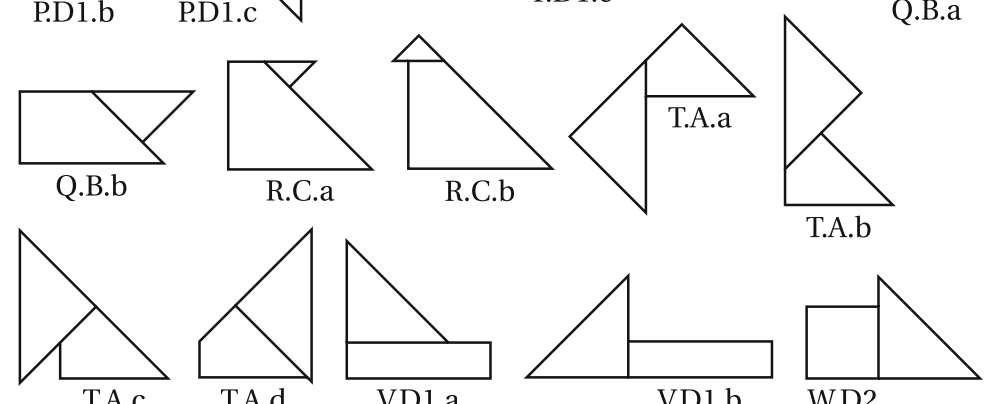

Fig. 10 All 31 non-convex pentagonal non-lattice tangrams

$\{3311,3131,3221,3212,2222\}$ w.l.o.g. (cf. Sect. 4.2). Since the vertices and sides of $T_{1}$ are arranged along $\mathbb{Z}^{2}$, it is easy to find all possible candidates that are composed by $7,8,12,14$ or 15 basic triangles. Figure 9 depicts only those of them who can be tiled by a subfamily of all seven tans, because the others cannot represent $T_{1}$. Quadrangles with dotted dissections will not give rise to pentagonal tangrams in the end.

Finally, we consider every remaining candidate for $T_{1}$, which is either triangle D1 (or D2) from Fig. 8 or a quadrangle from Fig. 9. We pick a triangle of the respective size from Fig. 8 as a candidate $T_{2}^{\prime}$ for the shape of $T_{2}$. We check if both $T_{1}$ and $T_{2}^{\prime}$ can be tiled simultaneously by all seven tans. This excludes the quadrangles $\mathrm{H}, \mathrm{I}, \mathrm{N}, \mathrm{O}, \mathrm{S}$, $\mathrm{U}, \mathrm{Y}$ and $\mathrm{Z}$. If the tiling is possible, we choose all images $T_{2}$ of $T_{2}^{\prime}$ under a rotation by an odd multiple of $\frac{\pi}{4}$ such that $T_{1}$ together with $T_{2}$ forms a dissection of a pentagon $T$. The last is impossible for the quadrangle $\mathrm{X}$. This way we obtain the 31 mutually incongruent pentagons $T$ from Fig. 10. Respective dissections can be adopted from Figs. 8 and 9. The dissections are not unique in general. 
Proposition 16 There exist, up to isometry, exactly 31 non-convex simple pentagons that are non-lattice tangrams.

The characterization of all simple pentagonal tangrams is complete.

Acknowledgements Both authors express their gratitude to Dr. Carsten Müller for sharing his enthusiasm, posing problems and encouraging work on tangrams.

Funding Open Access funding provided by Projekt DEAL.

Open Access This article is licensed under a Creative Commons Attribution 4.0 International License, which permits use, sharing, adaptation, distribution and reproduction in any medium or format, as long as you give appropriate credit to the original author(s) and the source, provide a link to the Creative Commons licence, and indicate if changes were made. The images or other third party material in this article are included in the article's Creative Commons licence, unless indicated otherwise in a credit line to the material. If material is not included in the article's Creative Commons licence and your intended use is not permitted by statutory regulation or exceeds the permitted use, you will need to obtain permission directly from the copyright holder. To view a copy of this licence, visit http://creativecommons.org/licenses/by/4.0/.

\section{References}

Brunner, C.: Symmetrische Elfecke mit dem Tangramspiel (project work that participated in the competition "Schüler experimentieren") (2014)

Brunner, C.: Teile und herrsche! Quadratzerlegung mit dem Tangram (project work that participated in the competition "Schüler experimentieren") (2015)

Elffers, J.: Tangram. Das alte chinesische Formenspiel. DuMont, Köln (1978)

Gardner, M.: More on tangrams: Combinatorial problems and the game possibilities of snug tangrams. Sci. Am. 231(3), 187-191 (1974)

Gardner, M.: Some new and dramatic demonstrations of number theorems with playing cards. Sci. Am. 231(5), 122-125 (1974)

Goodrich, A.T. (ed.): The new and fashionable Chinese puzzle. Printed for and published by A.T. Goodrich \& Co. no. 124 Broadway, opposite the City-Hotel, New-York. Also published by them, a key, wherein is explained the method of forming every figure contained in that pleasing amusement. Copy-right secured. New York (1817)

Graber, R.B., Pollard, S., Read, R.C.: Star tangrams. Recreat. Math. Mag. 2016(5), 47-60 (2016)

Heinert, D.: Konkave Fünfecke beim Tangramspiel (project work that participated in the competition "Schüler experimentieren") (1996)

Heinert, D.: Langweiliges Legespiel oder mathematische Herausforderung (I). Die WURZEL. Zeitschrift für Mathematik, Jahrgang, 1998(7), 159-166 (1998a)

Heinert, D.: Langweiliges Legespiel oder mathematische Herausforderung (II). Die WURZEL. Zeitschrift für Mathematik, Jahrgang, 1998(8), 180-187 (1998b)

Müller, C.: TANGRAM 1-4. (privately published) (1997-2014)

Müller, C.: 50 Jahre Spezi in Jena: Ein mathematischer Blick auf eine ganz SPEZIelle Schule. BoD—Books on Demand (2013)

Müller, C.: Tangram. 25 Jahre auf den Spuren der sieben Teile des Siebenschlau. BoD—Books on Demand (2020)

Pohl, S.S.: Gittertangramme mit maximalem Durchmesser (project work that participated in the competition "Jugend forscht") (2018)

Pohl, S.S.: Die Menge der Tangramfünfecke ist entschlüsselt—ein Problem von 1942 ist gelöst (project work that participated in the competition "Jugend forscht") (2019)

Pohl, S.S.: Die Menge der einfach nicht konvexen Tangramme (project work that participated in the competition "Jugend forscht") (2020)

Read, R.C.: Tangram. 330 Legespiele. Hugendubel, München (1985)

Read, R.C.: The snug tangram number and some other contributions to the corpus of mathematical trivia. Bull. Inst. Combin. Appl. 40, 31-39 (2004) 
Slocum, J., Botermans, J., Gebhardt, D., Ma, M., Ma, X., Raizer, H., Sonneveld, D.: The Tangram Book. The story of the Chinese puzzle. With over 2000 Puzzles to Solve. Sterling, New York (2004)

Wang, F.T., Hsiung, C.-C.: A theorem on the tangram. Am. Math. Mon. 49(9), 596-599 (1942)

Publisher's Note Springer Nature remains neutral with regard to jurisdictional claims in published maps and institutional affiliations. 